\title{
PIV Study of the Flow Across the Meridional Plane of Rotating Cylinders with Wide Gap
}

\author{
David S. Adebayo, Aldo Rona
}

\begin{abstract}
This study investigates by Particle Image Velocimetry (PIV) the wavy Taylor vortex flow that develops in the gap between concentric rotating cylinders of low aspect ratio and low radius ratio, which is a configuration relevant to oil bearing chambers in turbomachinery. The configuration is characterised by a larger annular gap width than in classical journal bearing and by a Taylor number (Ta) of $2.47 \times 10^{6}$, which is 1000 times higher than the first critical Taylor number. It is found that the non-uniform axial spacing of the vortex cores induces an asymmetric radial velocity profile and an inflected axial velocity profile close to the cylindrical walls. The outflow at the centre of each pair of Taylor vortices that make up a vortex cell is strong and it is characterised by a sharp radial velocity peak. The inflow between pairs of Taylor vortex cells is unconventional in that it is characterised by pairs of confluent streams from the outer cylinder that merge towards the inner cylinder. These results form a body of experimental evidence on the flow features that may occur in engineering practice in oil bearing chamber flows, with relevance to lubrication and wear. Copyright $(2015$ Praise Worthy Prize S.R.l. - All rights reserved.
\end{abstract}

Keywords: Particle Image Velocimetry, Concentric Cylinders, Wide Gap, Taylor Vortices, Meridional Plane PIV, High Taylor Number Flow

\section{Introduction}

The study of the flow in a wide gap between concentric rotating cylinders stands to benefit important engineering applications such as lubrication oil bearing chambers in gas turbines for aerospace propulsion and in the solvent extraction process industry.

The appearance and the development of flow instabilities between rotating concentric cylinders was first studied experimentally and analytically by Taylor [1].

Since the time Taylor [1] established the threshold at which a steady Couette flow becomes unstable, several studies explored the onset of this instability in differently rotating cylinders by modelling and experimentation.

The main non-dimensional parameters that determine this threshold are the Taylor number, or the equivalent Reynolds number, the ratio of the two radii $\eta=R_{i} / R_{o}$, and the aspect ratio $\Gamma=L\left(R_{o}-R_{i}\right)$.

The Taylor number:

$$
T a=\frac{2 \eta^{2} d^{4}}{1-\eta^{2}}\left(\frac{\Omega_{1}}{v}\right)^{2}
$$

and the equivalent Reynolds number

$$
R e=\frac{\Omega_{1} R_{i} d}{v}
$$

where $R_{i}$ and $R_{o}$ are the radii of the inner and outer cylinder respectively, $L$ is the length of the cylinders, $\Omega_{i}$ is the rotational speed of the inner cylinder, $d$ is the gap width, which is the distance between the outer diameter (OD) of the inner cylinder and the inner diameter (ID) of the outer cylinder, and $v$ is the fluid kinematic viscosity.

Based on these parameters, different flow states have been identified by [2]-[7] as the Taylor number increases beyond the initial threshold. The initial steady Couette flow changes to axisymmetric time-independent Taylor vortices (Taylor Vortex Flow) and subsequently to nonaxisymmetric unsteady time-dependent wavy Taylor vortices (Wavy Vortex Flow) [1]-[2], [8]-[10].

The non-axisymmetric unsteady time-dependent wavy Taylor vortices have been studied experimentally by [2], [8]-[9], [11]-[14]. Models of the flow dynamics have been developed by [15]-[18]. The simple geometry of two coaxial cylinders lends itself to analytical and numerical treatments of this problem. This has generated many contributions on the transition between the different flow regimes and the interested reader is referred to [19]-[21] for a review of this topic.

The Wavy Vortex Flow (WVF) by further increases in Taylor number is predicted to change to Modulated Wavy Vortex Flow (MWVF) and subsequently to Turbulent Taylor Vortex Flow (TTVF) [5].

Many of the early investigators on this flow instability focused their research on configurations with a radius ratio over the range $0.60 \leq \eta \leq 0.95$ and an aspect ratio 
$\Gamma \geq 20$, which is likely to have been driven by the modelling simplifications that can be used in the cases of small gap $(\eta \rightarrow 1)$ and large aspect ratio.

Adebayo and Rona [22] explored the flow regime at a high Taylor number in a flow configuration with the radius ratio over the range $0.40 \leq \eta \leq 0.60$ and aspect ratio over the range $7 \leq \Gamma \leq 12$. They documented by PIV the presence of an orderly flow with clearly defined time-mean wavy Taylor like vortex structures that persist over the Taylor number range $258 \times 10^{3} \leq \mathrm{Ta} \leq 10.93 \times$ $10^{6}$, where Modulated Wavy Vortex Flow or Turbulent Taylor Vortex Flow would have been expected based on the indications by Andereck et al. [5]. This orderly high Taylor number flow is substantially unexplored and the details of this flow in the meridional plane have not been addressed by previous work.

The present investigation wishes to provide a detailed experimental physics-based interpretation of the flow across the full meridional plane. The large gap width between the cylinders motivates detailing the axial and radial velocity profiles close to both the inner and outer cylinder surfaces, as well as at the cylinder gap mid-span, so that the radial variation in the trends is made explicit.

The large gap width is also likely to allow greater freedom in the location of the vortex centres.

This hypothesis is tested by identifying the vortex centre positions in the PIV vector maps. For this investigation, the authors have developed a bespoke PIV illumination setup that allows the acquisition of velocity vector maps over the full meridional plane. This arrangement is likely to be of broader interest to the PIV community.

Having established the presence of the Taylor vortex persistence by experimental observation in [22], this paper focuses on describing the details of the features of this flow. This paper does not address the mode staging process from Couette flow to this flow regime or from this flow regime to higher order instabilities, since this paper is about characterising the flow at this flow regime only. A more in-depth discussion on mode staging is outside the scope of this paper and the interested reader is referred to [21] for an insight into this topic.

\section{Experimental Facility}

Particle Image Velocimetry (PIV) is used to characterise the flow features emerging in the low radius ratio and low aspect ratio cylinder assembly, in which the inner cylinder is rotating and the outer cylinder is fixed.

\section{II.1. Test Rig and Instrumentation}

Figs. 1 show a schematic of the experimental setup and of the instrumentation. The test rig centrepiece consists of a rotating inner cylinder and a stationary outer cylinder.

The flow is illuminated by a laser sheet, imaged by a CCD camera, and analysed by the Dantec Dynamic
Studio PIV software version 2.30.

The main test rig specifications for this experimental study are summarised in Table I and a more extensive descriptions of the experimental rig is given in [22].

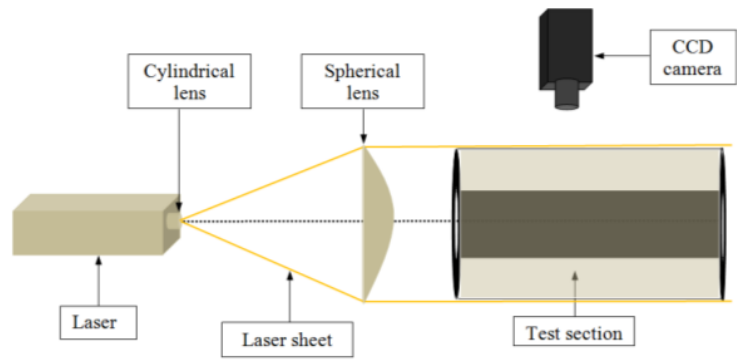

(a)

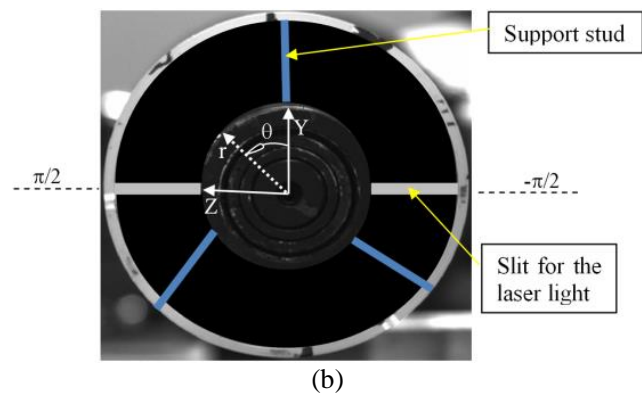

Figs. 1. (a) PIV experimental arrangement and (b) view from the laser side

TABLE I GEOMETRY OF THE TEST CASE

\begin{tabular}{cc}
\hline \hline Component & Dimension $(\mathrm{m})$ \\
\hline Inner cylinder length & 0.5 \\
Outer cylinder length & 0.52 \\
Inner cylinder outer diameter & 0.05 \\
Outer cylinder inner diameter & 0.114 \\
Gap width & 0.032 \\
\hline \hline
\end{tabular}

The layout of the PIV equipment used for this study consists of three sections as shown in Fig. 1(a). The laser sheet creation section comprises of a two-cavity doublepulsed Nd:YAG Litron L Nano laser and of the laser sheet optics. A trigger rate of $4 \mathrm{~Hz}$ is used to generate the light sheet.

A plano-convex spherical lens with an anti-reflective coating is positioned between the laser and the test section as shown in Fig. 1(a) to enable concurrent PIV measurements in the rectangular areas of illuminated flow either side of the inner cylinder. This makes the PIV setup unique in the context of the PIV application to Taylor vortex flows.

A Dantec Dynamics Studio seeding generator, model $10 \mathrm{~F} 03$, is used to atomize a mixture of $20 \%$ polyethylene glycol (PEG600) and 80\% water to produce seeding particles. The flow is imaged by a Dantec Dynamic Studio FlowSense 4M CCD camera that is placed at a right angle to the light sheet, as shown in Fig. 1(a).

The resolution of the camera is $2048 \times 2048$ pixels with a $60 \mathrm{~mm}$ AF Micro Nikon image lens, operated at a frequency of $4 \mathrm{~Hz}$ with an f/4 aperture. The CCD camera resolution of $2048 \times 2048$ pixels with a field of view of 
$250 \mathrm{~mm}$ gives a spatial resolution of $0.122 \mathrm{~mm} /$ pixel.

The adaptive correlation algorithm of DantecDynamics Studio PIV software version 2.30 was used for the cross-correlation during image processing of raw particle image pairs. The initial starting interrogation area was $32 \times 32$ pixel and ending with $16 \times 16$ pixel interrogation areas with a $50 \%$ overlap. Validation algorithms were applied to the raw velocity vector map to detect and remove erroneous vectors and produce a validated vector map.

\section{II.2. Experimental Procedure}

PIV measurements for the test case specified in Table I are conducted along a $0.25 \mathrm{~m}$ axial length of annulus between the rotating inner cylinder and the stationary outer cylinder.

The field of view and the non-dimensional flow parameters for the test case are summarised in Table II and in Table III respectively.

TABLE II

EXPERIMENTAL FIELD OF VIEW AREA

\begin{tabular}{cc}
\hline \hline Item & Value \\
\hline Axial length of the view area & $0 \leq X / R_{i} \leq 10$ \\
Radial length of the view area & $-2.28 \leq r / R_{i} \leq 2.28$ \\
Aspect ratio, $\Gamma$ & 7.81 \\
Radius ratio, $\eta$ & 0.44 \\
Clearance ratio & 1.28 \\
\hline \hline
\end{tabular}

TABLE III

FLOW TEST CONDITIONS

\begin{tabular}{cc}
\multicolumn{2}{c}{ Item } \\
\hline \hline Angular speed & $500 \mathrm{rpm}$ \\
Tangential speed & $1.31 \mathrm{~m} / \mathrm{s}$ \\
Taylor number, $\mathrm{Ta}$ & $6.47 \times 10^{6}$ \\
Reynolds number, $\mathrm{Re}$ & $2.87 \times 10^{3}$ \\
Ambient pressure & $759.5 \mathrm{mmHg}$ \\
Ambient temperature & $22.8^{\circ} \mathrm{C}$ \\
\hline \hline
\end{tabular}

Measurements are taken as the inner cylinder is rotated at a constant angular speed $\Omega_{\mathrm{i}}$ of $500 \mathrm{rpm}$. The corresponding inner cylinder tangential speed is detailed in Table III.

The Taylor number, aspect ratio and radius ratio fall within the parameter space associated to the orderly wavy Taylor like vortex structures identified by [22].

The average room temperature during the experiments was $22.5 \pm 0.5{ }^{\circ} \mathrm{C}$. Since the temperature excursion during the experiments was $\pm 0.5{ }^{\circ} \mathrm{C}$, the results have not been corrected for thermal effects.

The seeding particles in the test section were illuminated by a light sheet of approximately $1 \mathrm{~mm}$ thickness shone through the slit as shown in Fig. 1. One hundred image pairs were acquired. The time interval between the two frames was $1000 \mu \mathrm{s}$.

The statistical processing of the ensemble of 100 instantaneous velocity fields, data validation, removal of erroneous data, replacement of removed data, and data smoothing was carried out using Dantec Dynamic Studio software.

The convergence of the PIV data has been assessed by testing whether a certain number of PIV raw particle image pairs were enough to obtain a vector map average with a low statistical uncertainty.

By averaging between 100 and 120 images, the mean relative uncertainty in the PIV velocity was found to become nearly constant at approximately 5.5\%, suggesting that taking more PIV data was unlikely to change this result significantly. Based on this result, the PIV mean velocity fields obtained from averaging 100 instantaneous vector maps were taken as statistically converged.

\section{PIV Results and Discussion}

\section{III.1. Characteristics of the Flow Regime}

This paper is concerned with documenting closely the detailed flow features of the flow regime observed across the meridional plane of the coaxial rotating cylinders with aspect ratio $\Gamma=7.81$ and radius ratio $\eta=0.44$ tested at the high Taylor number $\mathrm{Ta}=6.47 \times 10^{6}$. Specific attention is given to the fluid dynamics observable from the PIV measurement. All the velocity fields have been normalised by the inner cylinder tangential speed $\Omega_{1} R_{i}$. The axial length in the PIV results has been normalised by the inner cylinder radius $R_{i}$ for consistency with the view area normalisation reported in Table II.

Fig. 2 displays the normalised ensemble averaged velocity vector maps obtained from 100 pairs of PIV images in the meridional plane of the annulus. Fig. 2 shows that the ensemble averaged flow is dominated by a typical Taylor vortex pattern over the entire measurement area. As the inner cylinder is rotated at a high Taylor number, the centrifugal force due to the rotation of the inner cylinder is greater than the pressure gradient due to the stationary outer cylinder wall.

This imbalance between the two forces causes the vortex centres in Fig. 2 to be shifted towards the wall of the outer cylinder as the centrifugal force pulls the fluid away from the rotating inner cylinder.

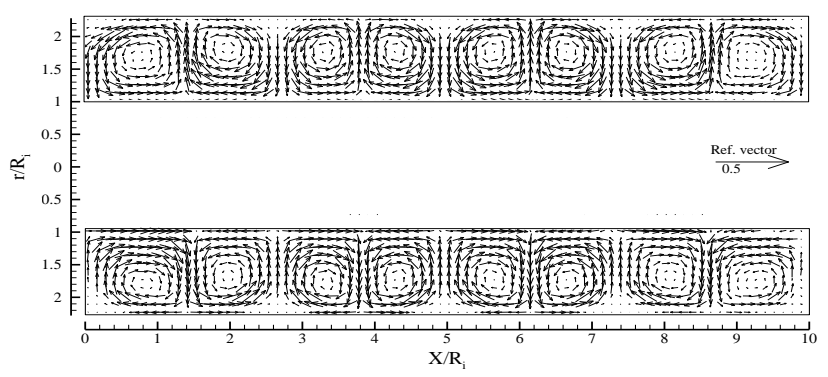

Fig. 2. Normalised time average of the velocity vectors from the PIV measurement in the meridional plane of the annulus, $\Gamma=7.81, \eta=$ 0.44 . The reference velocity vector is $0.5 \Omega_{1} R_{i}$ 
Along the axial direction of the PIV measurement view area, which is $10 R_{i}$ in length, the velocity field in Fig. 2 shows four pairs of vortices. The number of vortices was shown by [22] to be a function of the aspect and radius ratios at this wavy-like high Taylor number flow regime.

The radial motion induced by the Taylor vortices convects the fluid with high azimuthal momentum near the rotating inner cylinder radially outward, creating an outflow region between two adjacent vortices in each vortex pair. Similarly, low speed fluid near the stationary outer cylinder is convected radially inward, creating an inflow region between two adjacent pairs of vortices.

This redistributes the angular momentum of the fluid across the annulus. The consequent redistribution of flow mass across the annulus affects the inward flow and the outward flow velocity distribution. Thus, the radial outflow between the vortices is stronger than the radial inflow, as evidenced by the length of the velocity vectors in Fig. 2.

A distinct pattern of the flow features in the present study is the spatial oscillation of the Taylor vortices resulting in the radial and axial displacement of the vortex centres. The inward radial component of the velocity vectors at the end-walls is lower than that at the other inflow regions of the annulus, due to the wall shear stress at the left and right end walls. The amplitude of the spatial oscillation of the vortex centres is analysed in detail in the following section.

\section{III.2. Spatial Oscillation of the Vortex Centres}

The spatial oscillation of the vortex centres is analysed from the ensemble averaged velocity vector maps in Fig. 2. The vortex centres are defined in this paper as the locations where the meridional plane fluid velocity equals to zero in the annulus of the cylinder, excluding the wall boundaries where no-slip conditions apply.

In this paper, the coordinates of the Taylor vortex centres are determined by the cross-sectional lines algorithm described by [23], which is based on obtaining the difference between vortex induced velocity extrema.

The axial coordinate $X_{c}$ and the radial coordinate $r_{c}$ of each vortex centre are then used to indicate the centres of the vortices shown in Fig. 3. Fig. 3 confirms the streamwise variation in the radial positions of the Taylor vortex centres along the length of the cylinders identified in Fig. 2. In Fig. 3, the vortex centres of each Taylor vortex pair sit closer together than the vortex centres between neighbouring vortex pairs. This creates a difference in the vortex induced velocity.

Fig. 4 is used to illustrate the radial spread of the vortex centres in the meridional plane across both the lower and the upper channels $(\theta= \pm \pi / 2)$. In Fig. 4, open and filled symbols are used to identify the vortex centres at ( $\theta= \pm \pi / 2$ ). The abscissa in Fig. 4 has been normalised by the inner cylinder radius $R_{i}$, while the ordinate is normalised by the cylinder gap width $d$.
By comparing the vortex centres in the lower and upper channels in Fig. 4, a small variation in the axial position of the vortex centres between $\theta=\pi / 2$ and $\theta=-\pi / 2$ is noticeable. In Fig. 4, many of the vortices display a larger variation in the radial location of the vortex centres than the variation in their axial location.

This feature is noticeable both at $\theta=-\pi / 2$ and at $\theta=\pi / 2$. The radial variation is attributed to the flow regime at which this investigation was conducted and to the tolerance in setting the end walls orthogonal in the experimental arrangement. In [24], a conventional Taylor vortex flow was modelled with perfectly orthogonal end walls in a co-axial cylinder set-up at the same running conditions as this PIV experiment.

The numerical results predict a significantly smaller radial variation of the vortex centres. Similar results are obtained in the experimental and numerical modelling by [25]. The numerical viscosity that is intrinsic in the flow solver prevented the growth of secondary instabilities that the flow requires for staging to a higher Taylor mode, such as the wavy flow like structure observed in this PIV experiment. The numerical model of [24] predicted conventional Taylor vortices in the form of an array of counter-rotating vortex pairs with their vortex centres approximately aligned along the gap mid-span.

The greater radial and axial variation in $X_{c}$ and $r_{c}$ in this present work is therefore a feature associated with the flow regime in this test case.

The larger radial spacing near the end-wall is a consequence of wall boundary condition on the vortex centre distribution in addition to the flow regime type in this test case. These observations stand whether the vortex radial and axial coordinates are stated as dimensional or non-dimensional.

The horizontal dashed lines toward the top and bottom of Fig. 4 represent respectively the maximum and minimum limits of the spread band about the mean radial position of the vortex centres. The standard deviation of the radial position of the vortex centres was used to size the spread band, assuming a normal distribution. Based on the dimensions of the experimental domain, the normalised radial position between the inner and the outer cylinders is in the range $0 \leq\left(r-R_{i}\right) / d \leq 1$, which means the gap mid-span $\left(r=R_{i}+0.5 d\right)$ of the annulus is located at $\left(r-R_{i}\right) / d=0.5$. Fig. 4 shows that all vortex centres lie above the mid-gap $r=R_{i}+0.5 d$. In Fig. 4, the area occupied by the vortex centres spreads over the range $0.54 \leq\left(r-R_{i}\right) / d \leq 0.64$ radially and approximately $69 \%$ of the vortex centres is within the radial spread band, suggesting that there are a few outliers outside \pm 1 standard deviation band. This implies that the vortex position of the vortex centres changes as a function of time.

As such, the vortex centres are not symmetric about the mid-span of the annulus and do not coincide with the annulus gap mid-span. 


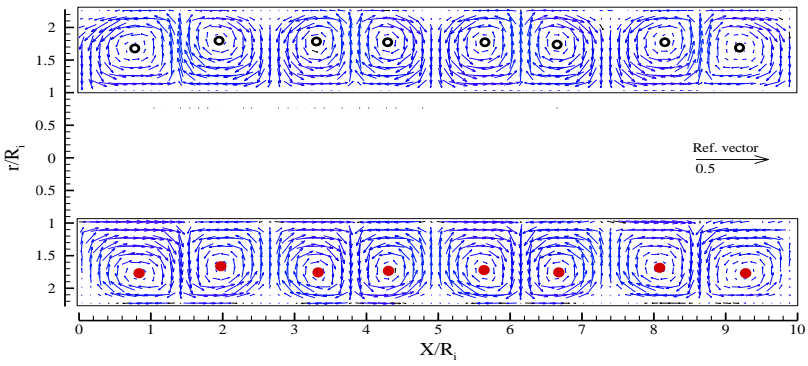

Fig. 3. Velocity vectors showing the centre of the vortices in open and filled circles on the meridional plane, $\Gamma=7.81, \eta=0.44$. The reference velocity vector is $0.5 \Omega_{1} R_{i}$

The vortex centres are more displaced toward the wall of the outer cylinder, due to the wide gap between the rotating inner cylinder and stationary outer cylinder in this test case.

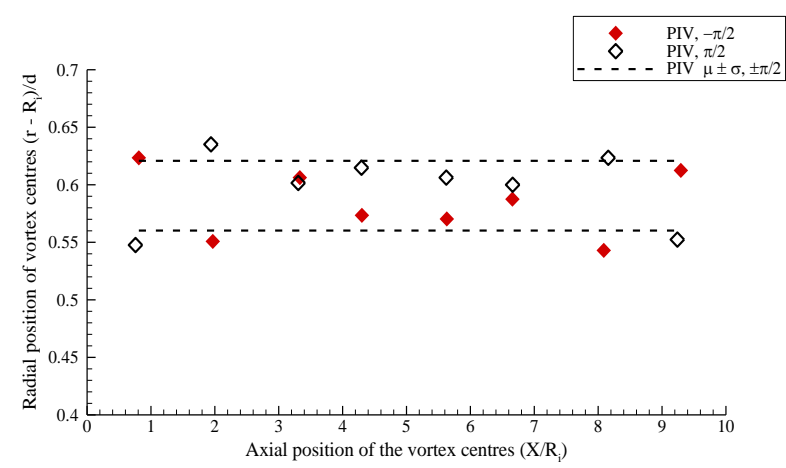

Fig. 4. Axial and radial position of vortex centres in the meridional plane with data spread bands about the vortex centre mean value from PIV, $\Gamma=7.81, \eta=0.44$.

\section{III.3. Axial Velocity Profiles}

The velocity profile along the gap mid-span of the annulus has been a focus of interest in most of the previous studies on the Taylor vortex flow including [26] and [27]. To the best of the authors' knowledge, no previous study has reported the velocity distribution at radial positions near the wall of the inner cylinder and near the wall of the outer cylinder.

The uniqueness of the large gap width between the cylinders in this study prompts detailing the axial and radial velocity profiles close to both the inner and outer cylinder surfaces. The radial velocity and axial velocity profiles at three different radial heights are therefore examined to understand in details the flow physics in these unexplored regions. The velocity profiles were extracted and plotted at constant radial positions along the axial direction on the lower $(\theta=-\pi / 2)$ channel of the annulus. The profiles are shown together with the PIV experimental error band, delimited by dashed lines, to document the experimental uncertainty.

The location of the vortex centres documented in Fig. 3 and Fig. 4 has a significant effect on the axial and radial velocity profiles.
In Fig. 5 and Fig. 6, the centres of the inward and outward flows are oblique saddle planes of axial velocity separating each Taylor vortex. The axial velocity profiles display alternating maxima and minima, with zero crossings in between, which is where the $r=R_{i}+0.5 d$ mid-span cylindrical surface intersects oblique saddle planes of axial velocity in the meridional plane.

The normalised axial velocity profile at $r=R_{i}+0.5 d$ (the gap mid-span of the annulus) and $\theta=-\pi / 2$ is shown in Fig. 5. Fig. 5 shows that axial velocity maxima occur close to the cores of clockwise vortices and axial velocity minima occur close to the cores of the anticlockwise vortices of Fig. 2. The speed range covered by the normalised axial velocity profiles at this radial position is relatively small compared with the surface speed of the rotating inner cylinder and the speed range of the axial velocity profiles at $r=R_{i}+0.125 d$ and $r=R_{i}+0.875 d$ in Fig. 6 .

The fact that the normalised axial velocity at $r=R_{i}+0.5 d$ is not zero in Fig. 5 at each vortex centre axial location is a consequence of the radial displacement of the vortex centres away from $r=R_{i}+0.5 d$ shown in Fig. 4.

Since the centres of the vortices do not lie along the $r=R_{i}+0.5 d$ line, there is a small axial velocity induced by the vortices at $r=R_{i}+0.5 d$.

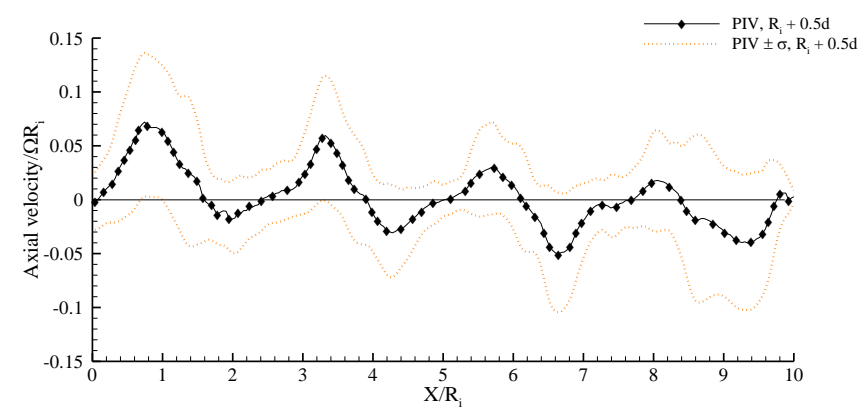

Fig. 5. Normalised axial velocity profiles in the meridional plane at the constant radial position $r=R_{i}+0.5 d$ with PIV error bands, $\theta=-\pi / 2, \Gamma=7.81, \eta=0.44$.

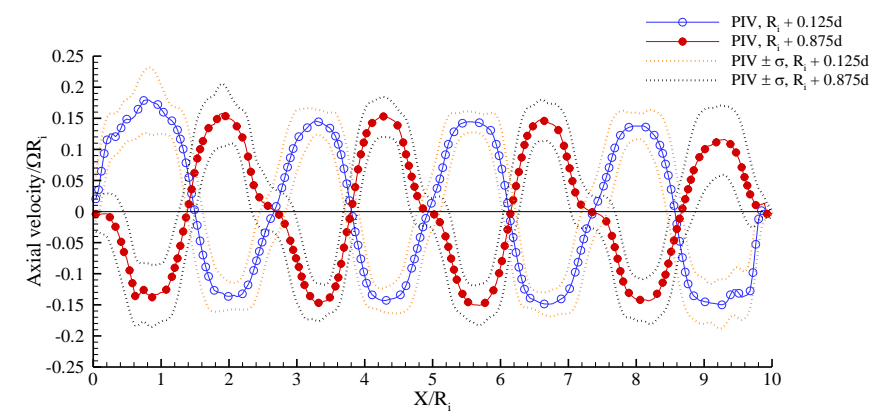

Fig. 6. Normalised axial velocity profiles in the meridional plane at constant radial positions $r=R_{i}+0.125 d$ and $r=R_{i}+0.875 d$ with PIV error bands, $\theta=-\pi / 2, \Gamma=7.81, \eta=0.44$. 
In Fig. 5, along the constant radial position $r=R_{i}+0.5 d$, the magnitude of the axial velocity at the right end-wall boundary, at $X=10 R_{i}$, is observed to be different from the one at the left end-wall at $X=0$.

This may be attributed to the difference in the radial location of the vortex centres and to the misalignment in the end-wall boundaries. That is, if the end-wall boundaries were not perfectly aligned perpendicular to the inner cylinder rotation axis, this would result in a periodic forcing of the flow.

Fig. 6 shows an interesting feature of the axial velocity distribution near the wall of the inner cylinder, at $r=R_{i}+0.125 d$, and near the wall of the outer cylinder, at $r=R_{i}+0.875 \mathrm{~d}$. The axial velocity is driven by the motion of the vortices. Each clockwise vortex induces an axial velocity maximum radially below its core at $r=R_{i}+0.125 d$ and a minimum radially above its core at $r=R_{i}+0.875 d$ at $\theta=-\pi / 2$.

Similarly, each anti-clockwise vortex induces an axial velocity minimum radially below its core at $r=R_{i}+0.125 d$ and a maximum radially above its core at $r=R_{i}+0.875 d$ and at $\theta=-\pi / 2$.

In Fig. 6, inflection points on the profiles of the normalised axial velocity are observed along the zero axial velocity line. The inflection points on the profiles in Fig. 6 correspond to the axial locations of the inflow regions in Fig. 2. These locations identify areas where low momentum fluid near the outer wall is convected inwardly by the radial inflow. The inflections may therefore be an indication of a non-monotonic shear layer across these inflow regions as a result of low azimuthal momentum convected from the stationary outer cylinder.

The difference in the magnitude between the axial velocity near the wall of the inner cylinder and that near the wall of the outer cylinder is observed to be small, indicating that there is a balance in the net axial flow along both walls.

\section{III.4. Radial Velocity Profiles}

Fig. 7 displays the normalised radial velocity profile at $r=R_{i}+0.5 d$, which has been the radial coordinate of choice for most of the radial velocity profiles reported in the open literature, such as the ones reported in [26]-[27]. The profile exhibits a periodic trend along the axial direction that is consistent with the one reported in previous work [26]-[27].

Approximately four cycles of a periodic oscillation along $X / R_{i}$ are shown in Fig. 7. The radial velocity is not symmetric about $u_{r}=0$, the zero ordinate of Fig. 7 .

This feature is a result of the displacement of the vortex centres in both the axial and radial directions discussed in [22].

The specific PIV setup detailed in section II and the comparatively wide gap $d$ of this investigation allows the acquisition and analysis of the radial velocity profiles near the cylindrical walls. These profiles have not been previously reported in the literature and represent the first experimental contribution to the analysis of the nearwall Taylor flow structures at wide gap by PIV.

Fig. 8 and Fig. 9 display respectively the normalised radial velocity profiles near the wall of the inner and outer cylinders at $r=R_{i}+0.125 d$ and at $r=R_{i}+0.875 d$.

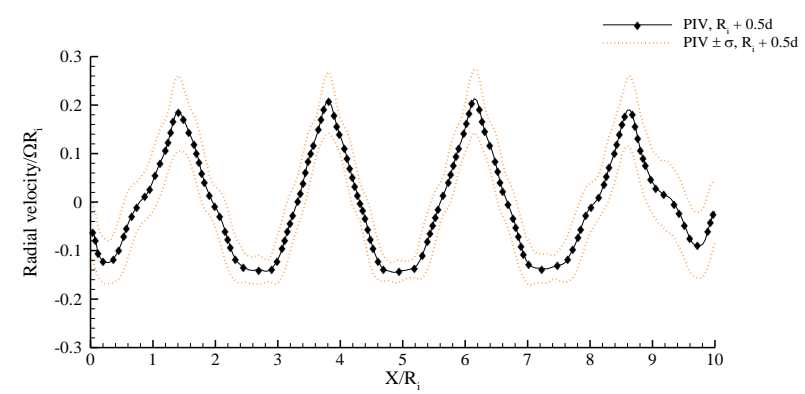

Fig. 7. Normalised radial velocity profiles in the meridional plane at constant radial position $r=R_{i}+0.5 d$ with PIV error bands, $\theta=-\pi / 2, \Gamma=7.81, \eta=0.44$

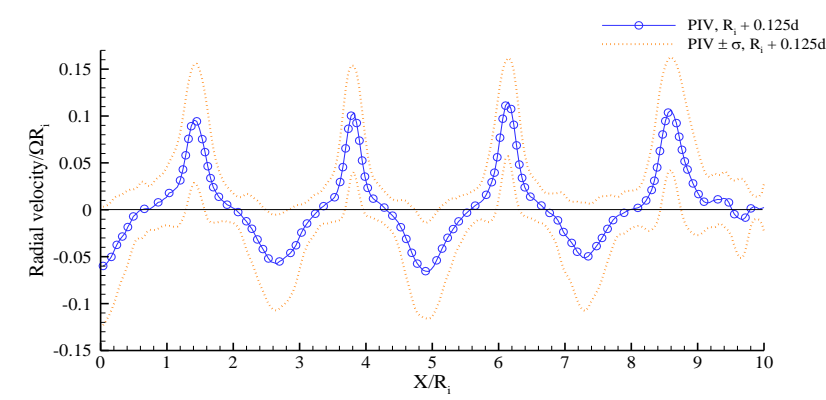

Fig. 8. Normalised radial velocity profiles in the meridional plane at constant radial position $r=R_{i}+0.125 d$ with PIV error bands, $\theta=-\pi / 2, \Gamma=7.81, \eta=0.44$

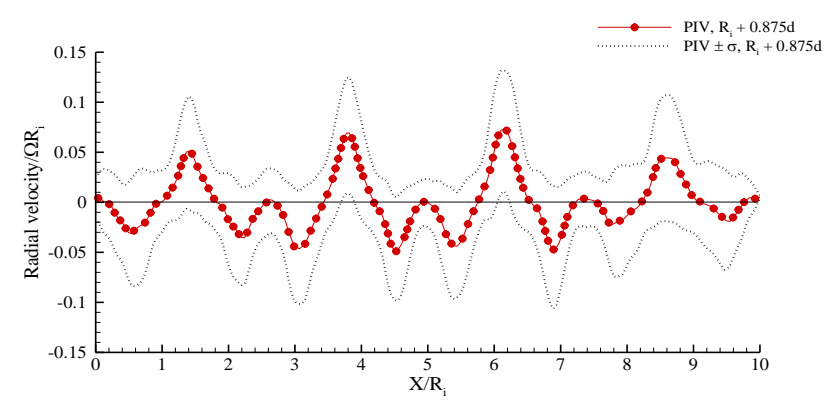

Fig. 9. Normalised radial velocity profiles in the meridional plane at constant radial position $r=R_{i}+0.875 d$ with PIV error bands, $\theta=-\pi / 2, \Gamma=7.81, \eta=0.44$

The locations of the radial velocity maxima and the radial velocity minima in Fig. 8 mark the meeting points of the radial outflow and radial inward flow respectively, between two adjacent vortices (induced downwells at $\theta=-\pi / 2$ on the meridional plane of Fig. 2 . 
The locations of the zero crossing of the radial velocity in Fig. 8 mark the centres of each vortex at $\theta=-\pi / 2$ on the meridional plane of Fig. 2 .

The radial velocity profiles in Fig. 8 exhibit the same periodic trend along the axial direction of Fig. 7.

These profiles also show that the radial velocity is not symmetric about the zero radial velocity line at $r=R_{i}+0.125 d$ and that the shape of the velocity peak at the outflow regions is sharper than that of the velocity trough at the inflow regions. This feature is attributed to the difference in the magnitudes of the Taylor vortex induced velocity at the axial velocity saddle planes between two adjacent vortices.

At these planes, the magnitude of the velocity vectors of the outflow regions is greater than the magnitude of the velocity vectors of the inflow regions, as shown in Fig. 2.

The sharper maxima (crests) in Fig. 8 correspond to jet-like outflows in the radial direction while the wider minima (valleys) correspond to radial sink-like inflows in Fig. 2.

The difference in the induced velocity is linked to the way in which the vortex centres of each Taylor vortex pair sit closer together than the vortex centres between neighbouring vortex pairs, as observed in Fig. 3.

The mass flow rate of the vortex pair induced outflow is balanced by the mass flow rate of the inflow between neighbouring vortex pairs, to satisfy mass conservation in the annular enclosure. Since the radial through-flow cross-sectional area between vortex pairs is smaller than the area between neighbouring vortex pairs, the outflow radial velocity is higher, generating the asymmetry observed in Fig. 8.

The radial velocity maxima and minima near the left end-wall of Fig. 8 are observed to be slightly different compared to the ones at the right end-wall. This feature is probably due to the difference in the boundary conditions at the two end-walls.

The discontinuous nature of the boundary conditions, where the inner cylinder meets the end-walls, generates weak circulation cells adjacent to the end-walls. As such, the radial velocity magnitude at both end-walls is lower than that at the other inflow region of the annulus. Also, the radial velocity at the left end-wall is affected by the presence of the slit for the laser light of Fig. 1(b).

In Fig. 9, at the outward flow regions, the normalised axial velocity profiles near the outer cylinder exhibit one single peak as the profiles near the inner cylinder.

The inward flow pattern in Fig. 9 is instead quite different, as the single trough minima of Fig. 8 are replaced by double trough minima in Fig. 9. This pattern can be explained by using simple potential flow theory. Consider any one vortex that has vortex neighbours on both sides in Fig. 3.

Let $r_{1}$ be the distance from the vortex centre in the meridional plane. The induced radial velocity $u_{r}$ of this vortex and its neighbour add so that, as the outflow saddle plane lies in the rotational portion of the vortex, where $u_{r} \propto r_{1}$, one radial velocity maximum is obtained.

The greater axial distance between the inflow saddle plane and the vortex centres from neighbouring Taylor vortex cells allows the saddle plane to lie in the portion of the vortex where $u_{r}$ decreases with $r_{1}$. This results in two distinct radial velocity minima in Fig. 9. This external vortex region correspond to the irrotational portion of a compound vortex in a potential flow, where $u_{r} \propto 1 / r_{1}$.

Each double trough minimum in Fig. 9 merges in a single wider minimum at $r=R_{i}+0.125 d$, close to the inner cylinder, as shown in Fig. 8. This shows that the induced velocity streams mix up by radial momentum transfer over the radial distance from $r=R_{i}+0.875 d$ to $r=R_{i}+0.125 d$, in the radial inflow.

These results have shown that the radial inflow is characterised by a relatively more complex flow pattern than the radial outflow.

The sharp maxima in Fig. 8 are axially aligned with the maxima in Fig. 9, indicating that the outflow shape is jet-like over a significant portion of the gap between the cylinders, over the range $R_{i}+0.125 d<r<R_{i}+0.875 d$.

The wider radial velocity minima in Fig. 8 are axially aligned with the double through minima of Fig. 9, indicating that there are two radially confluent streams merging in a single radial stream in the inflow regions.

This suggests that the flow between neighbouring vortex pairs is coupled to a less degree close to the outer cylinder than close to the inner cylinder. Such radial dependence of the degree of interaction between neighbouring Taylor vortex pairs may have practical implications for the development of successful models of the Taylor cell dynamics in wide gap configurations.

\section{Conclusion}

In this study, Particle Image Velocimetry was applied to the flow between concentric cylinders of radius ratio $\eta=0.44$, aspect ratio $\Gamma=7.81$, speed ratio $\mu=0$, and Taylor number $\mathrm{Ta}=6.47 \times 10^{6}$. Unlike the majority of the past investigations on the Taylor vortex flow that focused on large aspect ratio and low radius ratio configurations, this paper addressed the comparatively under-explored low aspect ratio and low radius ratio (wide gap width) configuration, relevant to important aerospace engineering applications such as lubrication bearing chambers of aircraft engines.

Previous investigations on Taylor instability have focused on either qualitative flow visualisations or quantitative measurements at the cylinder annulus midgap. By presenting PIV measurements on the meridional plane across the full annulus, this work documents previously unreported flow features in the annulus of coaxial rotating cylinders. These features mainly concern the inflow region between pairs of counter-rotating 
Taylor vortices. Inflection points are present in the profiles of normalised axial velocity near the wall of the outer cylinder, where the radial velocity profile exhibits double trough minima.

This represents two radially confluent streams merging in a single radial stream in the inflow regions, such that, close to the wall of the inner cylinder, the radial velocity profile is asymmetric and features a single wider minimum radially below each double through minima.

An approach for interpreting these features is proposed, based on potential flow concepts and on the observation that the axial distance between the inflow saddle plane and the vortex centres from neighbouring Taylor vortex cells is greater than that between the outflow saddle plane and the vortex centres.

This work has provided a body of experimental evidence that poses questions of both theoretical and practical nature. From the theoretical viewpoint, it challenges the flow dynamic modellers on whether these features are rendered by the current models. The presence of two confluent streams in the inflow region may indicate a degree of coupling between neighbouring vortex cells that is radially varying and perhaps lower towards the outer cylinder wall.

This may prompt further progress in modelling wavy Taylor vortex flow, leading to a more complete understanding of the flow dynamics.

The experimental evidence is of practical relevance to the design of oil bearing chambers. Specifically, the flow mixing between neighbouring vortex cells and possibly the heat transfer rate are likely to be lower than from a pattern of regularly spaced vortices in the axial direction.

This influences the migration of metal contaminants between cells. A single magnetic plug is often used to remove these particles, under the assumption that substantial flow mixing between cells takes place. This poses the question of how the newly observed flow features affect the effectiveness of this single point metal particle collection.

\section{Acknowledgements}

This project was supported by a Specific Targeted Research Project of the European Community's Sixth Framework Programme under project number 32669 (PROVAEN).

The experimental rig was fabricated and instrumented with help from Mr. Paul Williams, Thermofluids experimental officer, Mr. Dipak Raval and Mr. Julian Jones, engineering technicians, and the mechanical engineering workshop of the University of Leicester. The laser traverse was on loan from the Greenbank Group Ltd.

\section{REFERENCES}

[1] G. I. Taylor, Stability of a viscous liquid contained between two rotating cylinders, Philos. Trans. R. Soc. London A223 (1923),
289-343.

[2] D. Coles, Transition in circular Couette flow, Journal of Fluid Mechanics 21 (1965), 385-425.

[3] P. R. Fenstermacher et al, Dynamical instabilities and the transition to chaotic Taylor vortex flow, Journal of Fluid Mechanics 94 (1979), 103-129.

[4] R. W. Walden and R. J. Donnelly, Reemergent order of chaotic circular Couette flow, Physical Review Letters 42 (1979), 301 304.

[5] C. D. Andereck et al, Flow regimes in a circular Couette system with independently rotating cylinders, Journal of Fluid Mechanics 164 (1986), 155-183

[6] S. S. Deshmukh et al, Computational flow modeling and visualization in the annular region of annular centrifugal extractor, Industrial and Engineering Chemical Research 46 (2007), 8343-8354.

[7] S. S. Deshmukh et al, Flow visualization and three-dimensional CFD simulation of the annular region of an annular centrifugal extractor, Industrial and Engineering Chemical Research 47 (2008), 3677-3686.

[8] A. H. Nissan et al, The onset of different modes of instability for flow between rotating cylinders, Al. Ch. E. J. 9 (1963), 620-624.

[9] K. W. Schwarz et al, Modes of instability in spiral flow between rotating cylinders, Journal of Fluid Mechanics 20 (1964), 281 289.

[10] P. J. Gollub and H. L. Swinney, Onset of turbulence in rotating fluid, Physical Review Letters 35 (1975), 927-930.

[11] H. A. Snyder, Stability of rotating Couette flow. I. Asymmetric waveforms. Physics of Fluids 11 (1968), 728-734.

[12] M. Hetenyi and W. G. Vincenti, Proceedings of the 12th International Congress of Applied Mechanics, Stanford University, August 26-31 1968, (Springer, Berlin, Heidelberg, New York, 1968, pp 158-178).

[13] P. Castle et al, Visual observations and torque measurements in the Taylor vortex regime between eccentric rotating cylinders, ASME Journal of Lubrication Technology 93 (1970), 121-129.

[14] C. A. Jones The transition to wavy Taylor vortices, Journal of Fluid Mechanics 157 (1985), 135-162.

[15] R. C. Di Prima, Stability of non-rotationally symmetric disturbances for viscous flow between rotating cylinders, Physics of Fluids 4 (1961), 751-755.

[16] F. E. Bisshopp, Asymmetric inviscid modes of instability in Couette flow, Physics of Fluids 6 (1963), 212-217.

[17] R. J. Donnelly et al, Experiments on the stability of viscous flow between rotating cylinders. VI Finite-amplitude experiments, Proceedings of the Royal Society of London, A283 (1965), 531556.

[18] A. Davey et al, On the instability of Taylor vortices, Journal of Fluid Mechanics 31 (1968), 17-52.

[19] H. L. Swinney and J. P Gollub, Hydrodynamic instabilities and the transition to turbulence, second ed. (Springer-Verlag, New York, 1981, pp. 139-180).

[20] N. P. Cheremisinoff, Encyclopedia of Fluid Mechanics, (Gulf Publishing Company, Houston, 1985, pp. 237-273).

[21] E. L. Koschmieder, Benard cells and Taylor vortices, first ed. (Cambridge University Press, Cambridge, 1993).

[22] D. S. Adebayo and A. Rona, The persistence of vortex structures between rotating cylinders in the $10^{6}$ Taylor number range, (2015) International Review of Aerospace Engineering (IREASE), accepted for publication.

[23] H. Vollmers, Detection of vortices and quantitative evaluation of their main parameters from experimental velocity data, Measurement Science and Technology 12 (2001), 1199-1207.

[24] D. S. Adebayo, Annular flows and their interaction with a cylindrical probe, Ph.D. dissertation, Dept. Eng., University of Leicester, Leicester, UK, 2012.

[25] D. Deng, A numerical and experimental investigation of Taylor flow instabilities in narrow gaps and their relationship to turbulent flow in bearings, Ph.D. dissertation, The University of Akron, 2007.

[26] N. Abcha et al, Qualitative relation between reflected light intensity by Kalliroscope flakes and velocity field in the CouetteTaylor flow system, Experiments in Fluids 45 (2008), 85-94.

[27] R. Deng et al, Characterisation of Taylor vortex flow in a short 
liquid column. Journal of American Institute of Chemical Engineers 55 (2009), 3056-3065.

\section{Authors' information}

Department of Engineering, University of Leicester, LE1 7RH, UK.

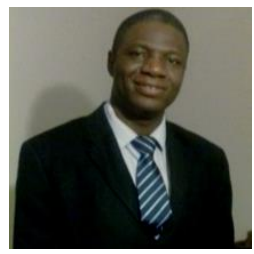

David S. Adebayo received a Bachelor of Engineering (BEng) degree from the University of Ilorin, Nigeria in 1992, a Master of Science in advanced mechanical engineering with distinction from the University of Leicester, Leicester, UK, in 2005 and a Ph.D. in engineering from the University of Leicester, Leicester, UK, in 2012.

Prior to his Master and Ph.D. degrees at the University of Leicester, he worked in many engineering industries in collaboration with international organisations for more than a decade and served in various capacities managing several projects. He has been Honorary Visiting Fellow of the University of Leicester since 2013 and teaching MEng aerospace engineering students. He has actively pursued research in annular flows and their interaction with a cylindrical probe, with application to aerospace engineering.

Dr. Adebayo is affiliated to the IMechE and a member of professional bodies: Nigeria Society of Engineers (NSE) and Council for the Regulation of Engineering (COREN).

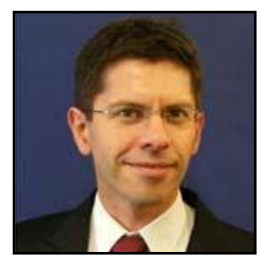

Aldo Rona received a Bachelor of Engineering in aeronautics from City University, London, UK, in 1993 and a Ph.D. in computational fluid dynamics of unsteady compressible flows from Southampton University, Southampton, UK, in 1997. As EPSRC Research Fellow at the Department of Aeronautics and Astronautics, University of Southampton, (1996-1997), he studied vortex boundary layer interactions by laser velocimetry. He developed time-resolved numerical models of unsteady transonic flows for high speed air transport applications as EU Marie Curie Fellow at the Department of Aeronautics and Aerospace, von Karman Institute for Fluid Dynamics, Belgium, in 1998. He joined the Department of Engineering at the University of Leicester, Leicester, UK, in 1998 as Lecturer where he has been working as Senior Lecturer since 2010. He has actively pursued research in unsteady flows relevant to air transport operations, with a focus on jets and jet noise, cavity flow and its contribution to airframe noise, and over-shroud leakage flow in axial turbines. Dr. Rona is a chartered engineer (CEng) and member of the Royal Aeronautical Society (MRAeS). 